\title{
THE DIAGNOSIS OF UVEITIS IN LEPROSY
}

by H. E. НоввS, F.R.C..S.

The incidence of blindness among patients afflicted with leprosy is unknown. The frequency of ocular involvement is found to vary in the different forms of the disease, from country to country and between separate communities in them. Reliable statistics appear only from the larger modern leprosaria where facilities for diagnosis and treatment are adequate and the proportion of cases with ocular complications from such centres is seen to be a good deallower than that found by many leprologists among isolated communities. The opinion of KIRWAN (1955) that, if the patient lives long enough and the disease persists, some form of ocular leprosy will eventually appear offers some explanation of these apparently conflicting views. Experience in a small leprosarium in England* can provide no figures likely to be applicable to the general problem of blindness from leprosy; but certain conclusions with regard to the diagnosis of the conditions leading to it may reliably be drawn from cases in all stages of the disease examined there over a period of ten years.

The ocular lesions chiefly concern the cornea and uveal tract. Their diagnosis presents peculiar problems and in the detection of the earliest stages that of uveitis-iritis, iridocyclitis-is probably the greater. At both sites the reactions of the eye to invasion by the leprosy bacillus appear to be diminished: pain is subdued through ocular anaesthesia from trigeminal involvement and the diagnostically-valuable injection of the outer eye is much less than would be expected in similar forms of disease produced by other organisms. Thus the onset of these conditions less readily attracts the attention of either patient or doctor and some degree of permanent visual loss has not infrequently occurred when the patient first presents for examination. In the case of the cornea, however, premonitory superficial keratitis may herald the onset of neuroparalytic keratitis and lagophthalmos gives warning of the further damage to be expected from the incomplete protection of the diseased cornea. The turbid aqueous humour which is the early sign of the common form of iritis, on the other hand, is visible only to instrumental inspection and signs which present to the naked eye are likely to be those of established complications. Treatment with atropine and cortisone drops from the moment of diagnosis is imperative and, if continued throughout the period of activity, can control the condition and prevent serious visual loss.

Chronic serous or plastic iritis is generally acknowledged by ophthalmologists familiar with leprotic lesions to be the commonest

* The Homes of St. Giles, East Hanningfield, Essex. 
manifestation of uveal involvement. Its onset is insidious and its course relentless for long periods. Inflammatory exudate from the iris and ciliary body passes into the aqueous and from it discrete deposits later adhere to the posterior corneal surface as keratic precipitates ('K'P.') or to the anterior lens capsule. Posterior synechiae form slowly; but may eventually occlude the pupil and result in secondary glaucoma. Nutrition of the lens, bathed in the pathological aqueous, is impaired and opacification frequently ensues. Disorganization of the globe with the appearance of gross staphylomata and phthisis bulbi are terminal events when vision has become extinct.

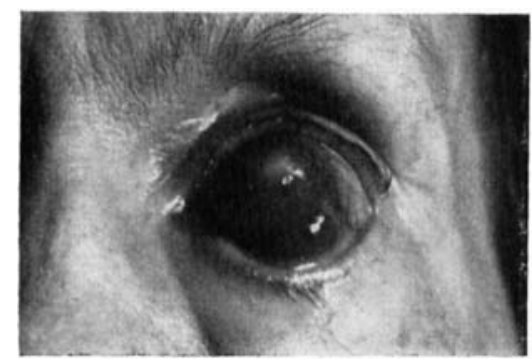

FIG. 1. Patient $H$. B. Staphyloma following prolonged, untreated uveitis in arrested lepromatous leprosy. Vision: No perception of light.

This sequence may also be seen following the appearance of 'iris pearls'-the deposits of lepra bacilli which so often seem to remain innocuous for long periods and which present one of the most fascinating pathological problems of the disease.

More rarely it begins with the appearance of leprosy nodules on the iris. The allergic type of serous iritis which is sometimes provoked in the course of the lepra reaction is more violent with more painful onset, greater ocular injection and a shorter, but no less visuallydisastrous course.

Detection of inflammatory exudates in the aqueous calls for examination under magnification and critical illumination, i.e., with the light-source focused upon the object.

Such optical conditions are most accurately provided in the slitlamp microscope, now considered an essential piece of equipment in every ophthalmic department. In its usual form it is, of course, 


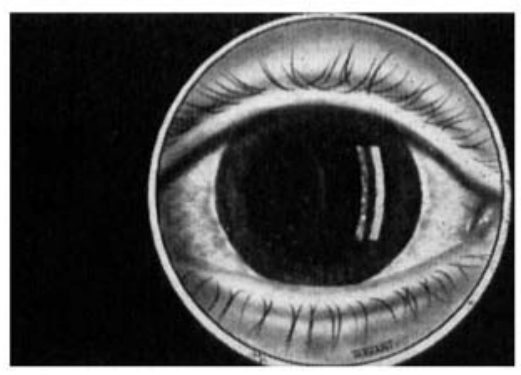

FIG. 2. Aqueous flare and keratic precipitates in active iridocyclitis as seen in the beam of the slit-lamp microscope.

static and thus accessible only to hospital patients. Professor Ida Mann's portable slit-lamp microscope offers a solution of this problem for the itinerant ophthalmologist; but it must be admitted that the biomicroscopic technique of co-ordination of illumination and microscope during the searching movements of both is an art not easily perfected by the occasional user of the instrument.

Similar, if less perfect, optical conditions may be produced with a loupe $(x 6$ or $x 8)$ and a torch which projects the image of the lamp

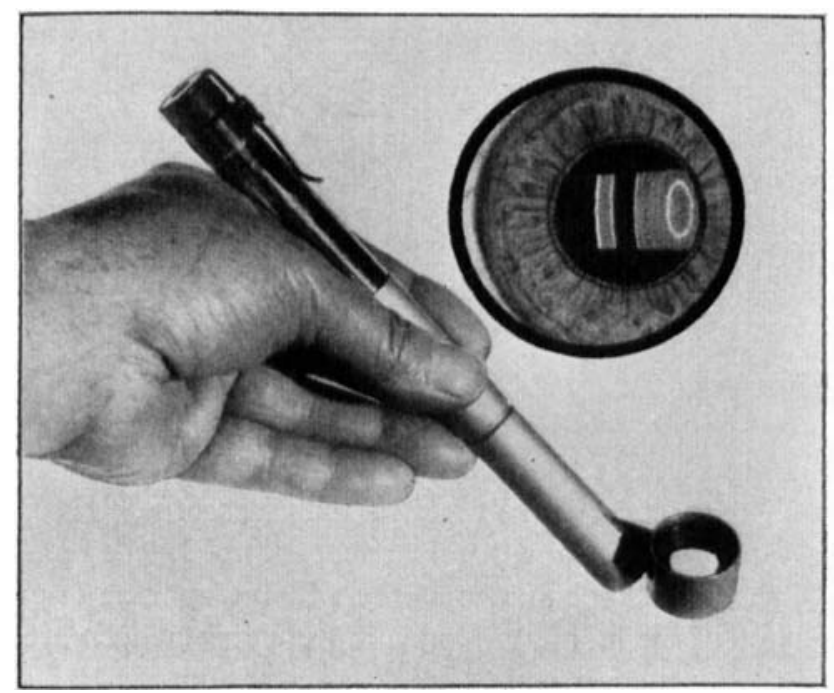

FIG. 3. The ilmluinated loupe and (inset) the optical section of the cornea, lens and anterior chamber as seen with it.

filament at its focus; but whilst these offer the desired degree of portability the bimanual technique of co-ordinating both whilst separating the eyelids with the fingers of one hand results in reliable 
observations only after constant practice. In order to simplify this technique a battery-operated illuminated loupe has been made for me.*

In this the light from a linear-filament bulb is condensed to a line which coincides with the focus of a $x 6$ loupe so that when the image of the eye under examination is brought into focus critical illumination is automatically achieved. As will be seen from the illustration this permits examination of a simple form of optical section of the transparent tissues of the -anterior segment of the eye comparable with that of the slit-lamp microscope. The accuracy of this, as of that of any form of loupe examination, is, of course, considerably less than that of biomicroscopy proper; but the detection of a moderate degree of aqueous flare and of any but fine 'K.P.' is quite feasible with it.

This small instrument, originally designed for the purposes of undergraduate instruction, is inexpensive and simple in use, being easily held in the fingers of one hand whilst those of the other separate the eyelids of the eye under examination. It has proved sufficiently reliable for serious clinical use and may be found by the leprologist in the field a useful tool for examination of the anterior segment of the eye.

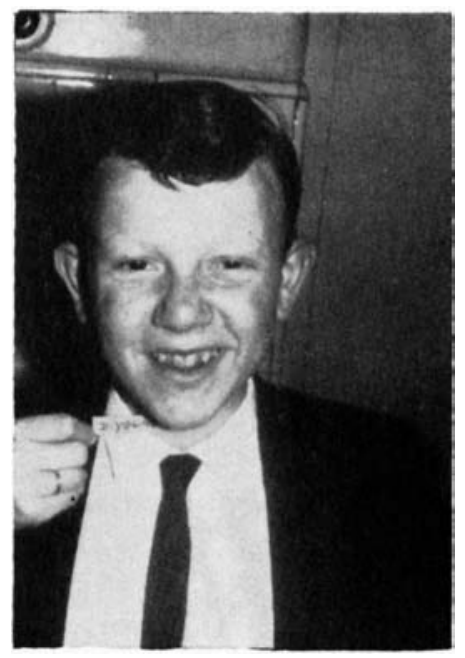

FIG. 4. Patient D. Y. Active lepromatous leprosy of six years' duration responding poorly to treatment. Bilateral uveitis presenting as aqueous flare during the first year and controlled by continuous treatment with atropine/cortisone drops. Vision, after five years, 6/6, in each eye and reading comfortable with glasses.

* By Stercks Martin, Ltd., New Cavendish Street, London, W.1. Price $£ 4$ 17s. 6d. 


\section{Acknowledgements}

I am grateful to Dr. R. G. Cochrane who first suggested the publication of this note and under whose care, also, the patients illustrated are. Figure 4 is from his photograph and figures 2 and 3 were produced for me by Dr. Peter Hansell of the Department of Medical Illustration at the Institute of Ophthalmology, London. The photograph in figure 1 was made by the Department of Medical Photography, The Royal Free Hospital, London.

\section{Reference}

Kirwan, E. W. O’G.: Proc. roy. Soc. Med. (1955), 48, 112. 